\title{
Supporting Information: The Electronic Spectrum of the Tropylium Cation in the Gas Phase
}

\author{
Ugo Jacovella, Michael S. Scholz, and Evan J. Bieske \\ School of Chemistry, The University of Melbourne, Victoria 3010, \\ Australia
}




\section{S1 Methods}

\section{S1.1 Theoretical methods and results}

The necessary input files, in order to replicate the calculations described in this work, are given in section 1.2.4.

\section{S1.1.1 Geometries and binding energies for $\mathbf{T r}^{+}, \mathbf{T r}^{+}$-Ar and $\mathrm{Bz}^{+}$}

Geometries and harmonic zero-point energies for $\mathrm{Tr}^{+}$, the $\mathrm{Tr}^{+}-\mathrm{Ar}$ complexes, and $\mathrm{Bz}^{+}$were calculated at the DFT PW6B95-D3(BJ)/jul-cc-pVTZ level of theory. ${ }^{1-3}$ The highest Abelian group symmetry was employed for the geometry optimizations. The electronic energies were refined with the DLPNO-CCSD(T) theory along with the aug-cc-pVQZ basis set. ${ }^{4,5}$ The "Superfine" integration grid was employed for the DFT computations. The DFT and DLPNO-CCSD(T) calculations were performed using the Gaussian 16 and ORCA 4.1 packages, respectively. ${ }^{6,7}$

Table S1: Bond lengths for $\mathrm{Tr}^{+}$in the $S_{0}$ and $S_{1}$ electronic states obtained from geometry optimization at CASSCF $(6,7) /$ def2-TZVP level of theory.

\begin{tabular}{lllll}
\hline Bond & State & Length $(\AA)$ & State & Length $(\AA)$ \\
\hline $\mathrm{C}-\mathrm{C}$ & $\mathrm{S}_{0}$ & 1.391 & $\mathrm{~S}_{1}$ & 1.404 \\
& & & & 1.415 \\
& & & & 1.425 \\
& & & & 1.428 \\
& & & & 1.426 \\
& & & & 1.417 \\
& & & & 1.406 \\
\hline $\mathrm{C}-\mathrm{H}$ & $\mathrm{S}_{0}$ & 1.074 & $\mathrm{~S}_{1}$ & 1.075 \\
& & & & 1.074 \\
& & & & 1.073 \\
& & & & 1.072 \\
& & & & 1.071 \\
& & & & 1.072 \\
& & & & \\
\hline
\end{tabular}


Table S2: Calculated vibrational frequencies in $\mathrm{cm}^{-1}$ for $\mathrm{Tr}^{+}$in the $\mathrm{S}_{0}$ and $\mathrm{S}_{1}$ states, determined at the CASSCF(6,7)/def2-TZVP level of theory. All vibrational frequencies are scaled by 0.915 , a factor that gives the most satisfactory match between simulated and experimental spectra and which is comparable to the factor used for similar systems. ${ }^{8}$ Each mode's symmetry is given for the $\mathrm{D}_{7 \mathrm{~h}}$ point group with corresponding symmetry upon descent to $C_{s}$ given in parentheses. 6 vibrational modes for the $\mathrm{S}_{1}$ state $\left(v_{5}, v_{9}, v_{15}\right)$ could not be clearly correlated with $\mathrm{S}_{0}$ state vibrations because of Duschinsky mixing.

\begin{tabular}{llcc}
\hline Mode & Symmetry & Frequency $\mathrm{S}_{0}$ state & Frequency $\mathrm{S}_{1}$ state \\
\hline$v_{1}$ & $\mathrm{a}_{1}{ }^{\prime}\left(\mathrm{a}^{\prime}\right)$ & 3079 & 3083 \\
$v_{2}$ & $\mathrm{a}_{1}{ }^{\prime}\left(\mathrm{a}^{\prime}\right)$ & 827 & 785 \\
$v_{3}$ & $\mathrm{a}_{2}{ }^{\prime}\left(\mathrm{a}^{\prime}\right)$ & 1428 & 1400 \\
$v_{4}$ & $\mathrm{a}_{2}{ }^{\prime \prime}\left(\mathrm{a}^{\prime \prime}\right)$ & 681 & 661 \\
$v_{5}$ & $\mathrm{e}_{1}{ }^{\prime}\left(\mathrm{a}^{\prime}\right)$ & 3075 & NA/NA \\
$v_{6}$ & $\mathrm{e}_{1}{ }^{\prime}\left(\mathrm{a}^{\prime}\right)$ & 1493 & $1441 / 1446$ \\
$v_{7}$ & $\mathrm{e}_{1}{ }^{\prime}\left(\mathrm{a}^{\prime}\right)$ & 964 & $696 / 870$ \\
$v_{8}$ & $\mathrm{e}_{1}{ }^{\prime \prime}\left(\mathrm{a}^{\prime \prime}\right)$ & 872 & $734 / 766$ \\
$v_{9}$ & $\mathrm{e}_{2}{ }^{\prime}\left(\mathrm{a}^{\prime}\right)$ & 3067 & NA/NA \\
$v_{10}$ & $\mathrm{e}_{2}{ }^{\prime}\left(\mathrm{a}^{\prime}\right)$ & 1593 & $1509 / 1528$ \\
$v_{11}$ & $\mathrm{e}_{2}{ }^{\prime}\left(\mathrm{a}^{\prime}\right)$ & 1211 & $1120 / 1143$ \\
$v_{12}$ & $\mathrm{e}_{2}{ }^{\prime}\left(\mathrm{a}^{\prime}\right)$ & 427 & $388 / 392$ \\
$v_{13}$ & $\mathrm{e}_{2}{ }^{\prime \prime}\left(\mathrm{a}^{\prime \prime}\right)$ & 1028 & $873 / 888$ \\
$v_{14}$ & $\mathrm{e}_{2}^{\prime \prime}\left(\mathrm{a}^{\prime \prime}\right)$ & 266 & $212 / 212$ \\
$v_{15}$ & $\mathrm{e}_{3}{ }^{\prime}\left(\mathrm{a}^{\prime}\right)$ & 3059 & NA/NA \\
$v_{16}$ & $\mathrm{e}_{3}{ }^{\prime}\left(\mathrm{a}^{\prime}\right)$ & 1511 & $1416 / 1433$ \\
$v_{17}$ & $\mathrm{e}_{3}{ }^{\prime}\left(\mathrm{a}^{\prime}\right)$ & 1279 & $1247 / 1250$ \\
$v_{18}$ & $\mathrm{e}_{3}{ }^{\prime}\left(\mathrm{a}^{\prime}\right)$ & 875 & $871 / 888$ \\
$v_{19}$ & $\mathrm{e}_{3}{ }^{\prime \prime}\left(\mathrm{a}^{\prime \prime}\right)$ & 1050 & $941 / 1010$ \\
$v_{20}$ & $\mathrm{e}_{3}{ }^{\prime \prime}\left(\mathrm{a}^{\prime \prime}\right)$ & 568 & $416 / 483$ \\
\hline & & &
\end{tabular}

\section{S1.1.2 Vertical excitation energy calculations}

Vertical excitation energies were computed using the equation-of-motion coupled cluster (EOM-CC) approach ${ }^{9}$ using the Gaussian 16 package. ${ }^{6}$ The cluster expansion was limited to single and double excitations (i.e. EOM-CCSD). The lowest two roots, corresponding to the $S_{1}$ and $S_{2}$ states, were calculated using the aug-cc-pVTZ basis set. ${ }^{5}$ The $1 s$ electrons on carbon atoms were left uncorrelated. The excitation energies were calculated using the $C_{2 v}$ Abelian subgroup of the ground-state point group $\mathrm{D}_{7 \mathrm{~h}}$. 


\section{S1.1.3 Franck-Condon/Herzberg-Teller simulations for the $S_{1} \leftarrow S_{0}$ spectrum of $\mathbf{T r}^{+}$}

The FCHT simulations were performed using the time-dependent implementation of de Souza, Neese, and Iszák. ${ }^{10}$

The input geometries and Hessian matrices for the $\mathrm{S}_{0} \mathrm{~A}_{1}^{\prime}$ and $\mathrm{S}_{1} \mathrm{E}_{3}^{\prime}$ states were calculated at the CASSCF(6,7)/def2-TZVP level of theory. ${ }^{11,12}$ The active space was comprised of the full $\pi$-system, i.e. six electrons distributed within seven orbitals. The def2-TZVP basis set was employed along with the def2/JK auxiliary basis for the resolution of the identity approximation applied to the CASSCF integral transformation steps. ${ }^{13}$ State-specific wavefunctions were computed for the $\mathrm{S}_{0}$ and $S_{1}$ states. Optimizations were performed using analytical gradients, whereas the Hessian matrices were computed via finite differences of analytical gradients with steps of $0.005 \mathrm{Bohr}$. Initial guess orbitals were generated from a restricted Hartree-Fock wavefunction with the def2-SV(P) basis. No symmetry constraints were employed for any CASSCF calculations.

Damping functions were employed for the dual purposes of phenomenologically modelling broadening in the experimental spectrum and to assist convergence of computing the correlation function. Linewidths of $12 \mathrm{~cm}^{-1}$ (Gaussian) and $100 \mathrm{~cm}^{-1}$ (Lorentzian) were used to compute "stick" and broadened spectra, respectively. Delta functions or narrower linewidth (i.e. longer decay time) damping functions were not practicable due to poor convergence in the correlation function. Spectra were simulated at $0 \mathrm{~K}$.

The lower $\left(\mathrm{S}_{0}\right)$ and upper state $\left(\mathrm{S}_{1}\right)$ geometries were superimposed to best satisfy the Eckart conditions and the normal modes were matched to take into account the Duschinsky rotation. The partial first derivatives of the transition dipole moment were calculated by finite differences with steps of 0.01 Bohr along the normal coordinates of the $S_{1} E_{3}^{\prime}$ state. Finally, the frequencies of the simulated spectrum were scaled by 0.915 and blue-shifted by $\sim 2990 \mathrm{~cm}^{-1}$ to best match the experimental spectrum.

All CASSCF and Franck-Condon/Herzberg-Teller calculations were performed using the ORCA 4.1 program. $^{7}$

\section{S1.1.4 $S_{1}$ potential energy surface for $\operatorname{Tr}^{+}$}

Potential energy surfaces for the $\mathrm{S}_{1}$ state were computed at the same $\operatorname{CASSCF}(6,7) /$ def2TZVP level of theory as the Franck-Condon/Herzberg-Teller calculations, with the addition of the RIJK approximation using the def2/JK auxiliary basis. ${ }^{13}$ The elec- 
tronic energy was calculated as a function of the $\mathrm{S}_{0} v_{2}$ and both degenerate components of the $S_{0} v_{7}$ normal coordinates starting from the ground state geometry (i.e. the Franck-Condon point). The scans were performed in dimensionless normal coordinates with a step size of 0.1 a.u. for both modes.

The state-averaged CASSCF wavefunction was computed including the $\mathrm{S}_{0}$ state and both components of the $S_{1}$ state at each grid point, yielding the adiabatic potential energy surfaces shown in Figs. $4 \mathrm{~b}$ and $5 \mathrm{~b}$.

\section{S1.1.5 Jahn-Teller vibronic coupling calculations}

In order to calculate the lowest vibronic levels and to estimate the impact of JT activity on the $S_{1} \leftarrow S_{0}$ electronic spectrum, the vibronic wavefunctions are modelled following the approach of Longuet-Higgins and coworkers. ${ }^{14,15}$ The vibronic wavefunctions are approximated as:

$$
\Psi=\psi^{+} e^{i \theta}+\psi^{-} e^{-i \theta}
$$

where $\psi^{+}$and $\psi^{-}$represent the diabatic wavefunctions before JT interaction and $\theta$ is the angular coordinate of the electronic state. The model Hamiltonian is given by a two-dimensional harmonic oscillator:

$$
\widehat{H}_{0}=\frac{1}{2}\left(r^{2}-\frac{\delta^{2}}{\delta r^{2}}-\frac{1}{r} \frac{\delta}{\delta r}-\frac{1}{r^{2}} \frac{\delta^{2}}{\delta \phi^{2}}\right)
$$

perturbed by a mixing operator:

$$
\widehat{H}^{\prime}=k r\left(e^{i(2 \theta-\phi)}+e^{-i(2 \theta-\phi)}\right)
$$

where $r$ and $\phi$ are the polar vibrational coordinates in terms of the two degenerate normal modes. $k$ is the dimensionless energy given by:

$$
k^{2}=\frac{2 E_{\mathrm{JT}}}{E_{\mathrm{ZPE}}}
$$

where $E_{\mathrm{JT}}$ is the JT stabilization (see Fig. 5c in the paper) and $E_{\mathrm{ZPE}}$ is the zero-point energy of the uncoupled doubly-degenerate $\omega_{\mathrm{JT}}$ (i.e. $E_{\mathrm{ZPE}}$ is $899 \mathrm{~cm}^{-1}$ ). Together, these equations give an expression for the vibronic energy levels:

$$
\left[\begin{array}{cc}
\widehat{H}_{0}-E & k r e^{-i \phi} \\
k r e^{i \phi} & \widehat{H}_{0}-E
\end{array}\right]\left[\begin{array}{l}
\psi_{l}^{+} \\
\psi_{l}^{-}
\end{array}\right]=0 .
$$


where the quantum number $l$ indicates the vibrational angular momentum. Following Longuet-Higgins and coworkers, ${ }^{14,15}$ the energy levels are numerically evaluated by expansion of $\psi_{l}^{+/-}$in a basis of isotropic 2-dimensional harmonic oscillators. The final eigenvalue problem for a given value of $l$ is given by:

$$
\left[\begin{array}{ccccc}
l+1-E & k \sqrt{l+1} & 0 & 0 & \ldots \\
k \sqrt{l+1} & l+2-E & k \sqrt{1} & 0 & \ldots \\
0 & k \sqrt{1} & l+3-E & k \sqrt{l+2} & 0 \\
0 & 0 & k \sqrt{l+2} & l+4-E & \ddots \\
\vdots & \vdots & 0 & \ddots & \ddots
\end{array}\right]\left[\begin{array}{c}
a_{1} \\
a_{2} \\
a_{3} \\
a_{4} \\
\vdots
\end{array}\right]=0 .
$$

Diagonalization of Equation S6 yields the vibronic energy levels for a given $l$ in units of $\omega_{\mathrm{JT}}$. The only parameter in this model is $k$, which is calculated using Equation S4 using the values of $E_{\mathrm{JT}}\left(120 \mathrm{~cm}^{-1}\right)$ and $\omega_{\mathrm{JT}}\left(899 \mathrm{~cm}^{-1}\right)$ extracted from the potential energy slices shown in Figure $5 \mathrm{c}$ in the manuscript. Diagonalization was performed with a basis of 1000 harmonic oscillator functions.

The energy levels are conveniently denoted by $\left|v_{7}, j\right\rangle$ where $v_{7}$ is the number of quanta in the $v_{7}$ mode, and $j=l \pm \Lambda / 2$, where $\Lambda$ is the quantum number associated with the projection of the total electronic angular momentum onto the principal axis. The $\mathrm{S}_{1}$ excited state has $l=-v, \ldots,-1,0,1, \ldots, v$ and $\Lambda=1$, giving $j= \pm 1 / 2, \pm 3 / 2, \ldots$. The selection rule for transitions between JT-active vibronic levels is $\Delta j= \pm 1 / 2$. The $v_{7}=0$ level of the $\mathrm{S}_{0}$ ground state has zero vibrational angular momentum $(l=0)$ and electronic angular momentum $(\Lambda=0)$, giving $j=0$. Therefore, transitions to the $S_{1}\left|v_{7} \geq 0, \pm 1 / 2\right\rangle$ vibronic levels are allowed and contribute to the spectrum, whereas transitions to $j= \pm 3 / 2, \pm 5 / 2, \ldots$ levels are forbidden.

The model of Longuet-Higgins and coworkers can be used to predict the intensities for transitions from the vibrationless level of the ${ }^{1} \mathrm{~A}_{1}^{\prime}$ ground state to the Jahn-Teller active vibrational levels of the ${ }^{1} \mathrm{E}_{3}^{\prime}$ state. ${ }^{14}$ Assuming equivalent harmonic vibrational frequencies in the $S_{1}$ and $S_{0}$ states, the intensities are given by the elementwise square of the eigenvectors from Equation S6. ${ }^{14,16}$ Relative intensities for transitions to the three lowest $j= \pm 1 / 2$ levels are predicted to be $1.00,0.21$, and 0.01 , respectively. In the case of dipole-allowed transitions for a range of other molecules it has been found that incorporating the intensities for the Jahn-Teller active modes from this model with the usual Franck-Condon intensities adequately reconstructs the experimental spectrum. ${ }^{16,17}$ 
Table S3: Jahn-Teller levels $\left|v_{7}, j\right\rangle$ for the $v_{7}$ mode of $\operatorname{Tr}^{+}$in the $\mathrm{S}_{1}$ state. Relative intensities of transitions to the $\left|v_{7}, 1 / 2\right\rangle$ levels from the $\mathrm{S}_{0} \mathrm{~A}_{1}^{\prime}$ ground state are also given.

\begin{tabular}{lll}
\hline level & energy & relative \\
$\left|v_{7}, j\right\rangle$ & $\left(\mathrm{cm}^{-1}\right)$ & intensity \\
\hline$|0, \pm 1 / 2\rangle$ & 805 & 1.0 \\
$|1, \pm 1 / 2\rangle$ & 1864 & 0.21 \\
$|2, \pm 1 / 2\rangle$ & 2650 & 0.013 \\
$|3, \pm 1 / 2\rangle$ & 3628 & 0.0007 \\
$|4, \pm 1 / 2\rangle$ & 4478 & 0.00002 \\
& & \\
$|1, \pm 3 / 2\rangle$ & 1550 & \\
$|2, \pm 3 / 2\rangle$ & 2837 & \\
$|3, \pm 3 / 2\rangle$ & 3497 & \\
$|4, \pm 3 / 2\rangle$ & 4550 & \\
& & \\
$|2, \pm 5 / 2\rangle$ & 2322 & \\
$|3, \pm 5 / 2\rangle$ & 3750 & \\
$|4, \pm 5 / 2\rangle$ & 4395 & \\
$|3, \pm 7 / 2\rangle$ & 3110 & \\
$|4, \pm 7 / 2\rangle$ & 4623 & \\
$|4, \pm 9 / 2\rangle$ & 3909 & \\
\hline
\end{tabular}




\section{S1.1.6 Input files for electronic structure calculations}

\section{DFT geometry optimization in Gaussian 16}

\begin{tabular}{|c|c|c|c|}
\hline \multicolumn{4}{|c|}{$\operatorname{tr}+. g j f-o p t i m i z a t i o n$ and frequencies for $\mathbf{T r}^{+}$} \\
\hline \multicolumn{4}{|c|}{$\%$ mem $=6 \mathrm{gb}$} \\
\hline \multicolumn{4}{|c|}{$\% \operatorname{chk}=\operatorname{tr} . \operatorname{chk}$} \\
\hline \multicolumn{4}{|c|}{ \# PW6B95D3/jul-cc-pVTZ opt=verytight freq int=superfine symm=loose } \\
\hline \multicolumn{4}{|c|}{ Tr+ optimization } \\
\hline \multicolumn{4}{|l|}{11} \\
\hline C & 0.00000000000000 & 1.60969400000000 & 0.00000000000000 \\
\hline C & 1.25850900000000 & 1.00362800000000 & 0.00000000000000 \\
\hline C & 1.56933600000000 & -0.35819100000000 & 0.00000000000000 \\
\hline C & -1.25850900000000 & 1.00362800000000 & 0.00000000000000 \\
\hline C & 0.69842000000000 & -1.45028400000000 & 0.00000000000000 \\
\hline C & -1.56933600000000 & -0.35819100000000 & 0.00000000000000 \\
\hline C & -0.69842000000000 & -1.45028400000000 & 0.00000000000000 \\
\hline $\mathrm{H}$ & 0.00000000000000 & 2.70350500000000 & 0.00000000000000 \\
\hline $\mathrm{H}$ & 2.11368500000000 & 1.68560800000000 & 0.00000000000000 \\
\hline $\mathrm{H}$ & 2.63572300000000 & -0.60158600000000 & 0.00000000000000 \\
\hline $\mathrm{H}$ & -2.11368500000000 & 1.68560800000000 & 0.00000000000000 \\
\hline $\mathrm{H}$ & 1.17300700000000 & -2.43577400000000 & 0.00000000000000 \\
\hline $\mathrm{H}$ & -2.63572300000000 & -0.60158600000000 & 0.00000000000000 \\
\hline $\mathrm{H}$ & -1.17300700000000 & -2.43577400000000 & 0.00000000000000 \\
\hline
\end{tabular}




bz+.gjf - optimization and frequencies for $\mathbf{B z}^{+}$
\%nprocshared=4
\%mem=6gb
\%chk=bz.chk
\# PW6B95D3/jul-cc-pVTZ opt=verytight freq int=superfine symm=loose
Bz+ optimization

1
1




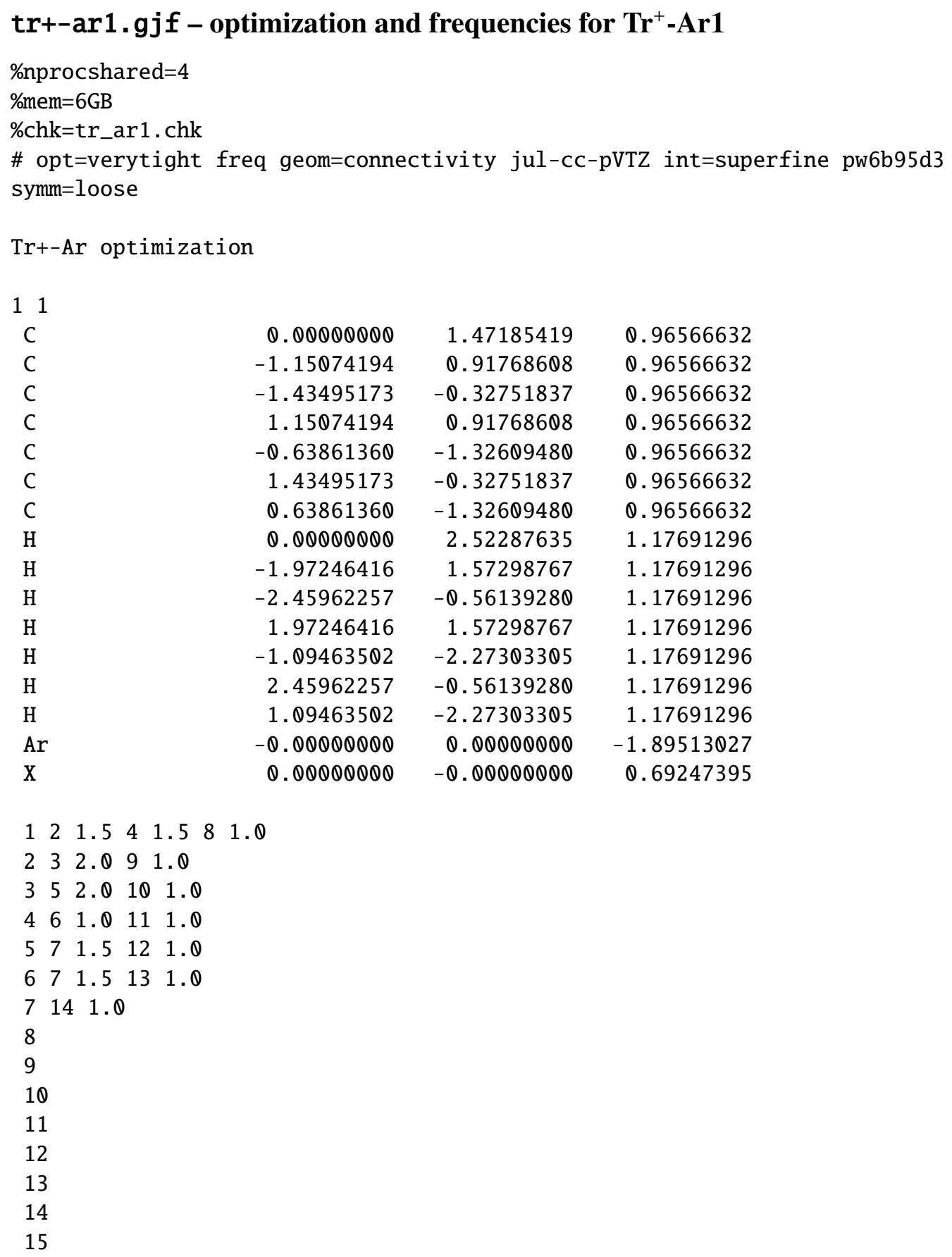

C

C

$\mathrm{H}$

$\mathrm{H}$

$\mathrm{H}$

$\mathrm{H}$

$\mathrm{H}$

$\mathrm{H}$

$\mathrm{H}$

Ar

$\mathrm{X}$

0.000000000
-1.15074194
-1.43495173
1.15074194
-0.63861360
1.43495173
0.63861360
0.00000000
-1.97246416
-2.45962257
1.97246416
-1.09463502
2.45962257
1.09463502
-0.00000000
0.00000000

1.47185419

0. 91768608

$-0.32751837$

0.91768608

$-1.32609480$

$-0.32751837$

$-1.32609480$

2. 52287635

1.57298767

$-0.56139280$

1. 57298767

$-2.27303305$

$-0.56139280$

$-2.27303305$

0.00000000

$-0.00000000$

0.96566632

0.96566632

0.96566632

0.96566632

0.96566632

0.96566632

0. 96566632

1.17691296

1.17691296

1.17691296

1.17691296

1.17691296

1. 17691296

1.17691296

$-1.89513027$

0.69247395

$\begin{array}{llllllll}1 & 2 & 1.5 & 4 & 1.5 & 8 & 1.0\end{array}$

$\begin{array}{llllll}2 & 3 & 2.0 & 9 & 1.0\end{array}$

$\begin{array}{llllll}3 & 5 & 2.0 & 10 & 1.0\end{array}$

$\begin{array}{llllll}4 & 6 & 1.0 & 11 & 1.0\end{array}$

$\begin{array}{llllll}5 & 7 & 1.5 & 12 & 1.0\end{array}$

$\begin{array}{lllll}6 & 7 & 1.5 & 13 & 1.0\end{array}$

7141.0

8

9

10

11

12

13

14

15 


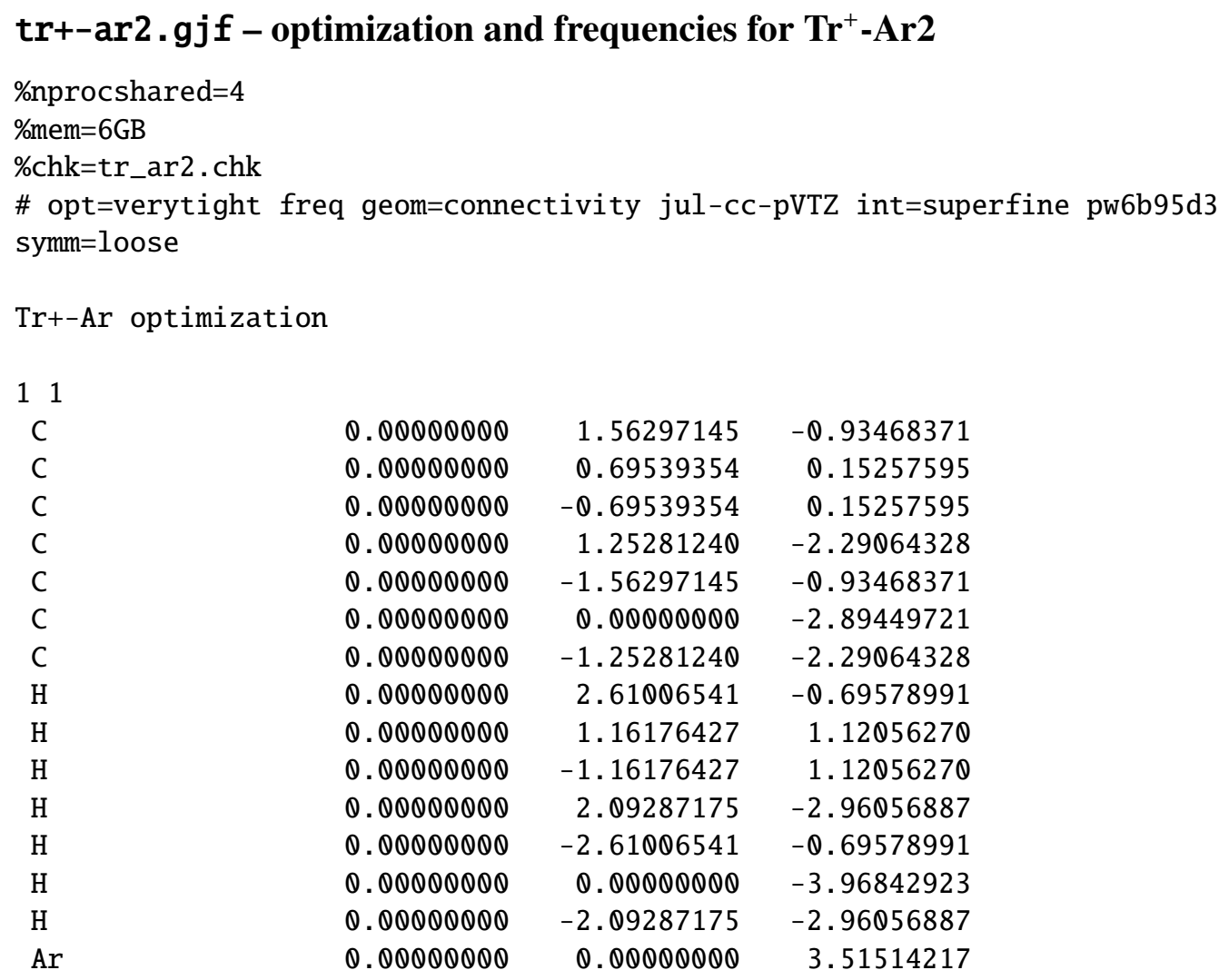

$\begin{array}{llllllll}1 & 2 & 1.5 & 4 & 1.5 & 8 & 1.0\end{array}$

$\begin{array}{lllll}2 & 3 & 1.5 & 9 & 1.0\end{array}$

$\begin{array}{lllll}3 & 5 & 1.5 & 10 & 1.0\end{array}$

$\begin{array}{lllll}4 & 6 & 1.5 & 11 & 1.0\end{array}$

$\begin{array}{lllll}5 & 7 & 1.5 & 12 & 1.0\end{array}$

$\begin{array}{lllll}6 & 7 & 1.5 & 13 & 1.0\end{array}$

$\begin{array}{llll}7 & 14 & 1.0\end{array}$

8

9

10

11

12

13

14

15 


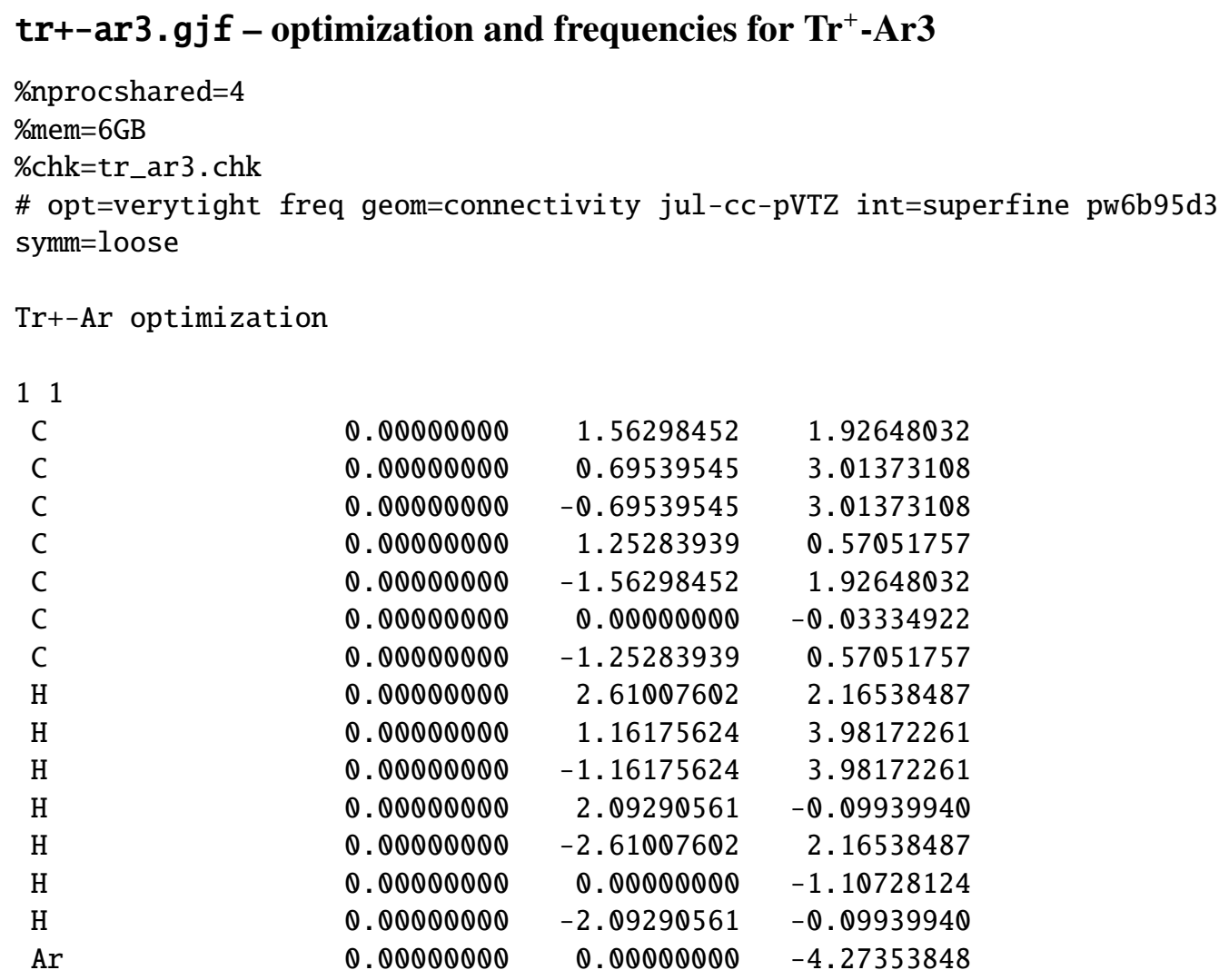

$\begin{array}{llllllll}1 & 2 & 1.5 & 4 & 1.5 & 8 & 1.0\end{array}$

$\begin{array}{lllll}2 & 3 & 1.5 & 9 & 1.0\end{array}$

$\begin{array}{lllll}3 & 5 & 1.5 & 10 & 1.0\end{array}$

$\begin{array}{lllll}4 & 6 & 1.5 & 11 & 1.0\end{array}$

$\begin{array}{lllll}5 & 7 & 1.5 & 12 & 1.0\end{array}$

$\begin{array}{lllll}6 & 7 & 1.5 & 13 & 1.0\end{array}$

7141.0

8

9

10

11

12

13

14

15 


\section{EOM-CCSD vertical excitation energies in Gaussian 16}

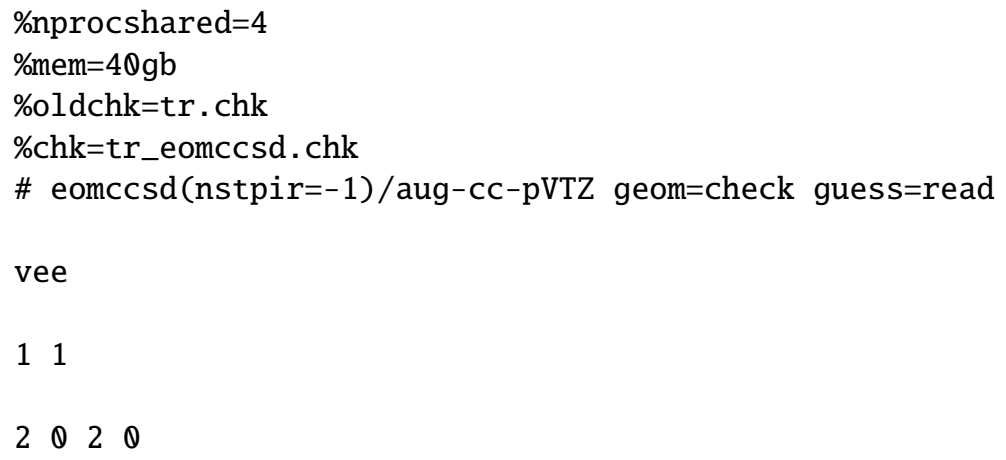




\section{DLPNO-CCSD(T) single-point energies in ORCA 4.1}

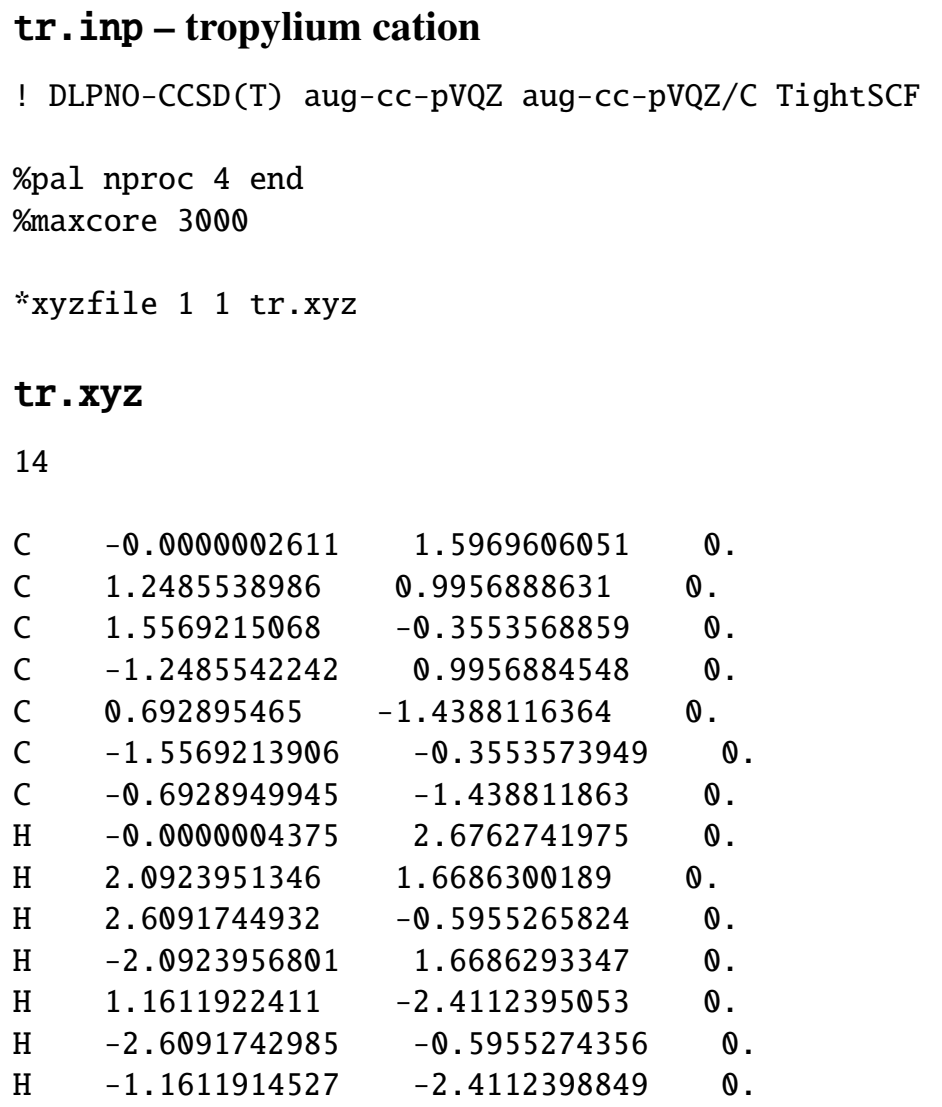




\begin{tabular}{|c|c|c|c|}
\hline \multicolumn{4}{|c|}{ bz. inp - benzylium cation } \\
\hline \multicolumn{4}{|c|}{ ! DLPNO-CCSD(T) aug-cc-pVQZ aug-cc-pVQZ/C TightSCF } \\
\hline \multicolumn{4}{|c|}{$\begin{array}{l}\text { \%pal nproc } 4 \text { end } \\
\% \text { maxcore } 3000\end{array}$} \\
\hline \multicolumn{4}{|c|}{ *xyzfile $11 \mathrm{bz} . x y z$} \\
\hline \multicolumn{4}{|c|}{ bz.xyz } \\
\hline \multicolumn{4}{|c|}{14} \\
\hline & 0.0000001873 & 0.0000013109 & -1.7760520842 \\
\hline & 0.0000078108 & -1.2260369396 & -1.1037772319 \\
\hline & 0.0000112764 & -1.2357755503 & 0.2587633232 \\
\hline$c$ & 0.0000070945 & -0.0000004901 & 0.9770066722 \\
\hline & -0.0000006915 & 1.2357755099 & 0.25876494 \\
\hline & -0.0000040628 & 1.2260386818 & -1.1037756279 \\
\hline$\Pi$ & -0.0000025198 & 0.0000020167 & -2.8550817025 \\
\hline$\Pi$ & 0.0000108556 & -2.1450461097 & -1.6639091652 \\
\hline n & 0.0000171452 & -2.1610828767 & 0.812093725 \\
\hline $\mathrm{H}$ & -0.0000037838 & 2.1610821123 & 0.8120965524 \\
\hline $\mathrm{H}$ & -0.0000099182 & 2.1450485847 & -1.6639063587 \\
\hline$c$ & 0.0000105018 & -0.0000013785 & 2.3351068095 \\
\hline & 0.0000163752 & -0.9221677611 & 2.8963409863 \\
\hline & 0.0000074445 & 0.9221642699 & 2.8963421928 \\
\hline
\end{tabular}




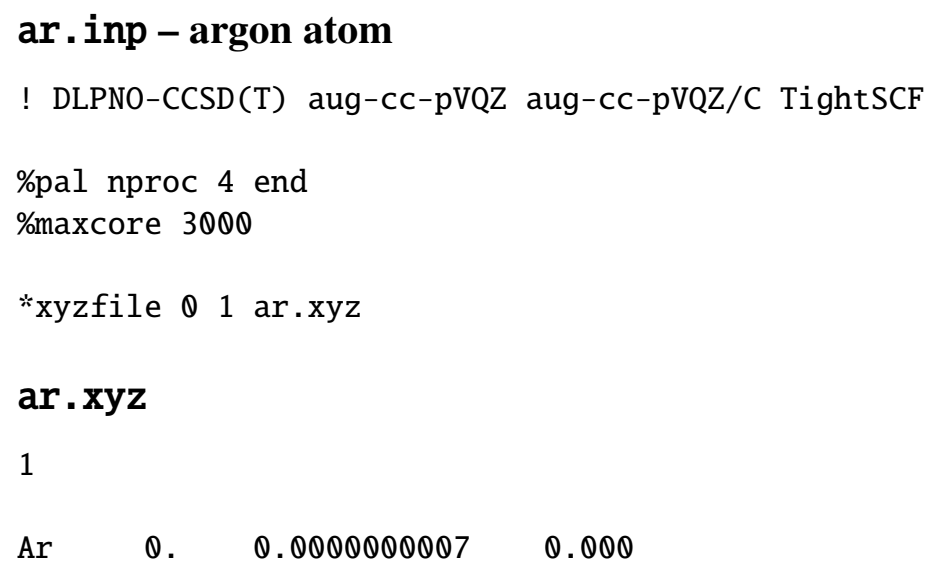




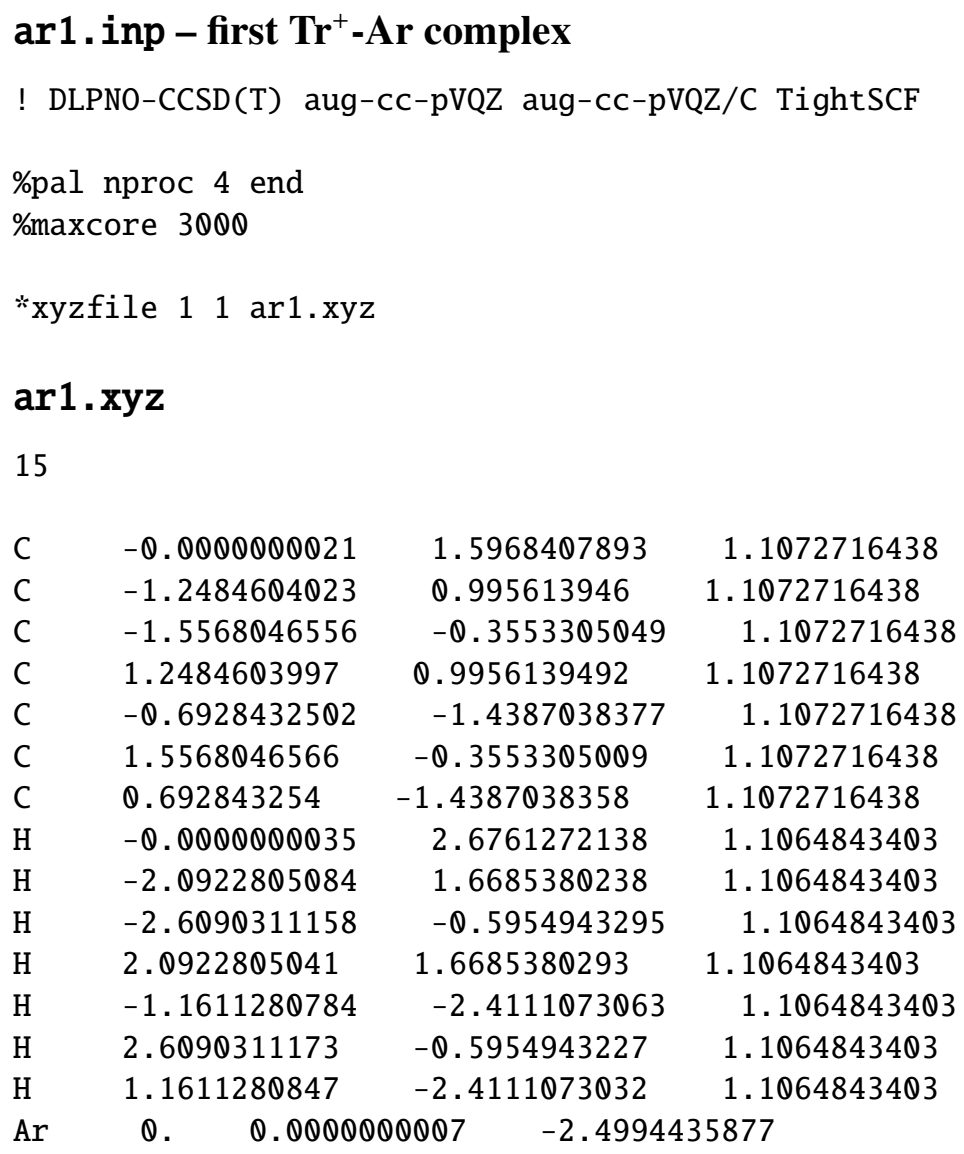




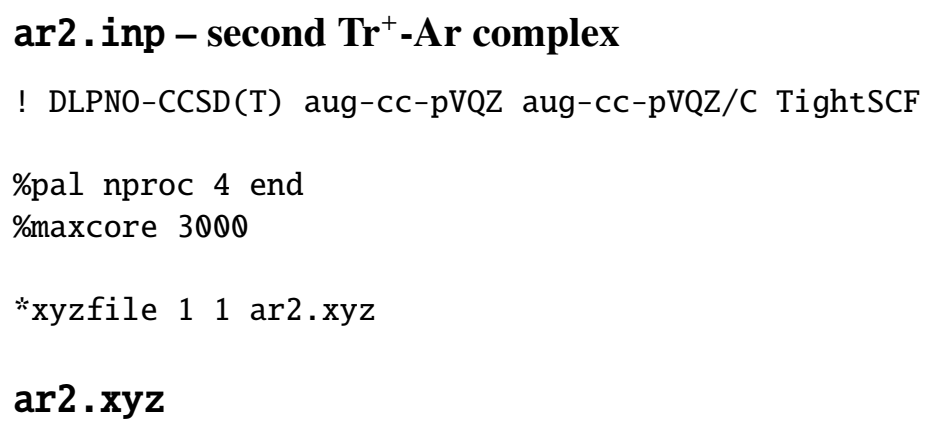




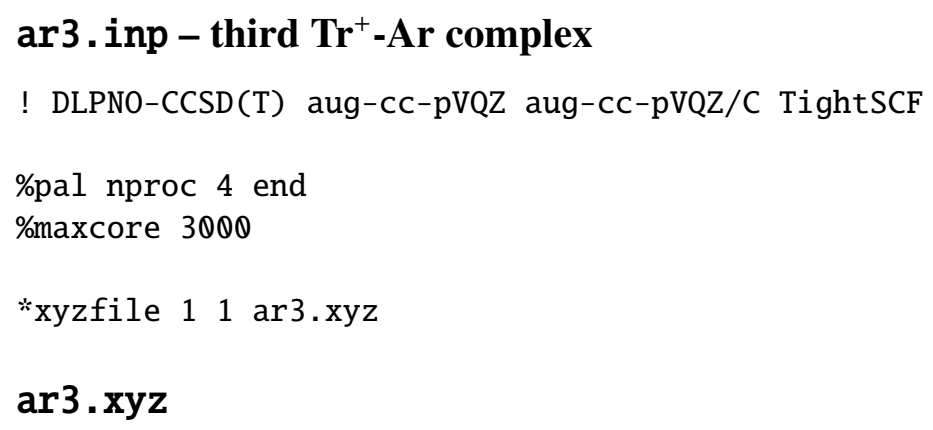




\begin{tabular}{|c|c|c|c|}
\hline \multirow{2}{*}{\multicolumn{4}{|c|}{$\begin{array}{l}\text { Franck-Condon/Herzberg-Teller simulations in } \\
\text { tr_guessorbs . inp - guess orbitals for CASSCF }\end{array}$}} \\
\hline & & & \\
\hline \multicolumn{4}{|c|}{ ! RIJK Conv def2-SV(P) def2/JK XYZFile } \\
\hline \multicolumn{4}{|c|}{ \%output } \\
\hline & \\
\hline \multirow{2}{*}{\multicolumn{3}{|c|}{ end $P$ Print[P_MOs] 1}} & \\
\hline & & & \\
\hline \multicolumn{4}{|c|}{$\%$ maxcore 1500} \\
\hline \multicolumn{4}{|c|}{ \%pal nproc 4 end } \\
\hline \multicolumn{4}{|c|}{ *xyz 11} \\
\hline 6 & 0.000000 & 1.609694 & 0.000000 \\
\hline 6 & 1.258509 & 1.003628 & 0.000000 \\
\hline 6 & 1.569336 & -0.358191 & 0.000000 \\
\hline 6 & -1.258509 & 1.003628 & 0.000000 \\
\hline 6 & 0.698420 & -1.450284 & 0.000000 \\
\hline 6 & -1.569336 & -0.358191 & 0.000000 \\
\hline 6 & -0.698420 & -1.450284 & 0.000000 \\
\hline 1 & 0.000000 & 2.703505 & 0.000000 \\
\hline 1 & 2.113685 & 1.685608 & 0.000000 \\
\hline 1 & 2.635723 & -0.601586 & 0.000000 \\
\hline 1 & -2.113685 & 1.685608 & 0.000000 \\
\hline 1 & 1.173007 & -2.435774 & 0.000000 \\
\hline 1 & -2.635723 & -0.601586 & 0.000000 \\
\hline 1 & -1.173007 & -2.435774 & 0.000000 \\
\hline
\end{tabular}




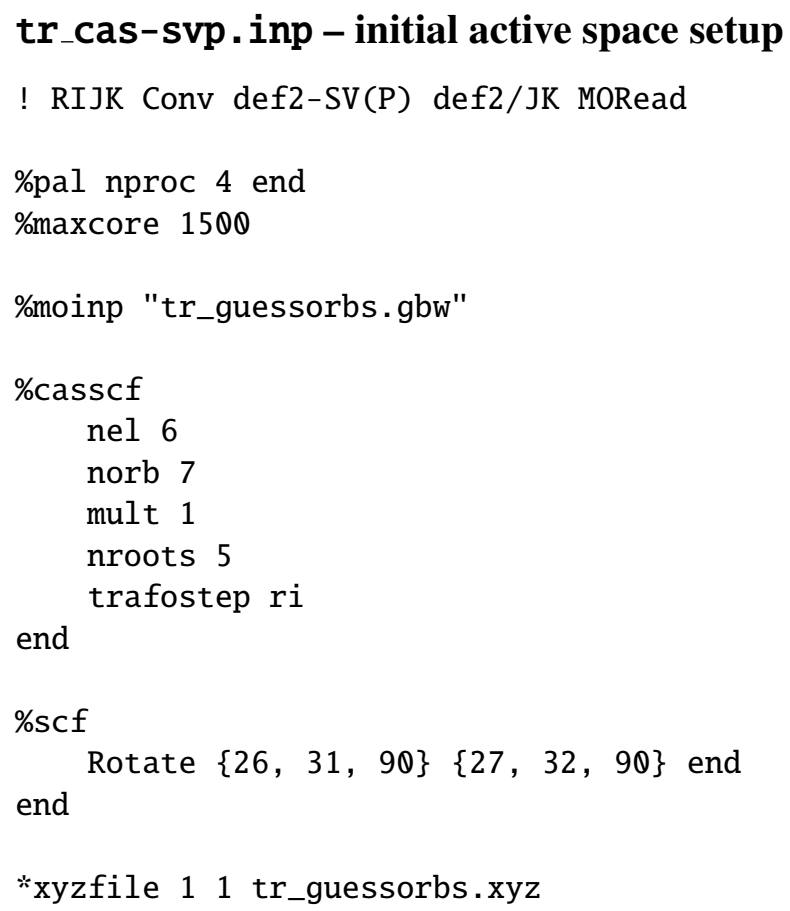




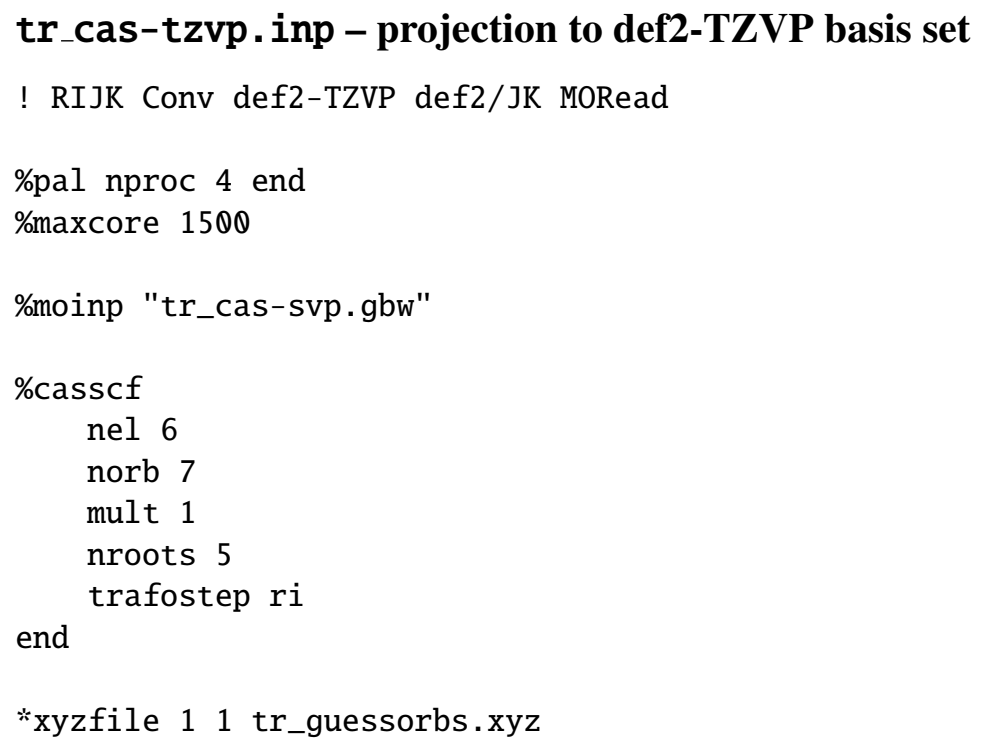




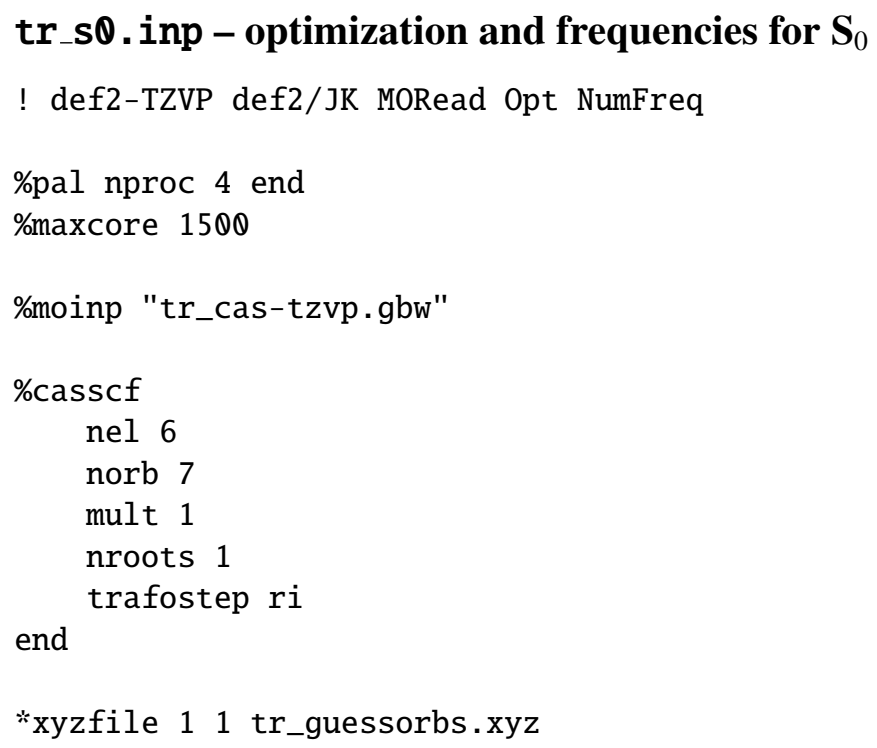




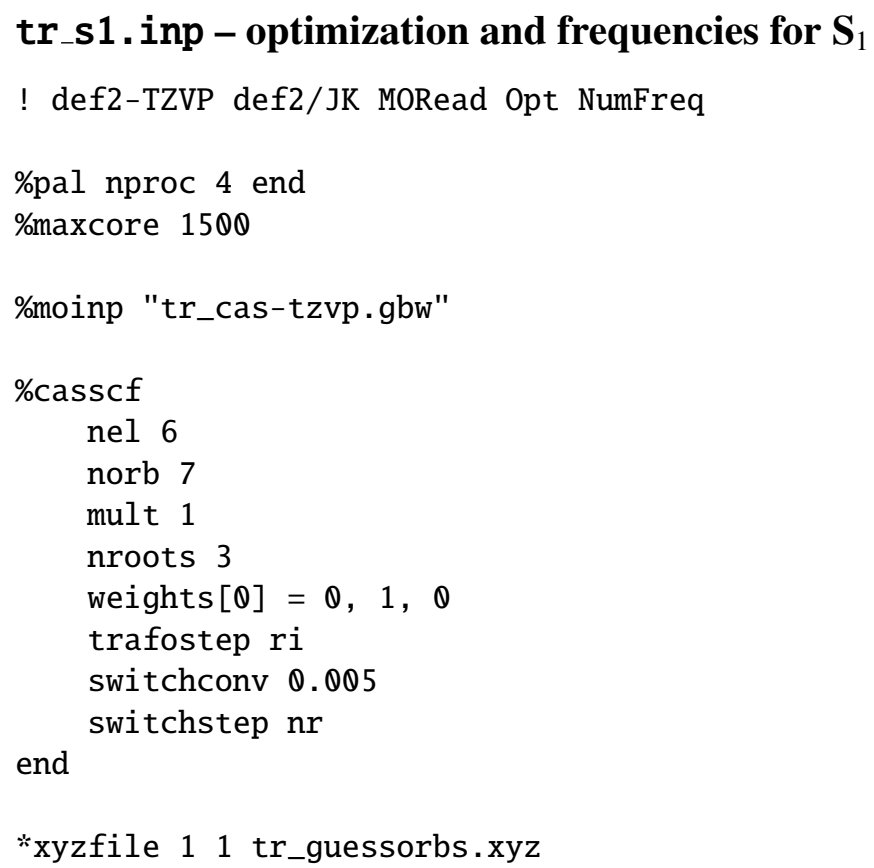




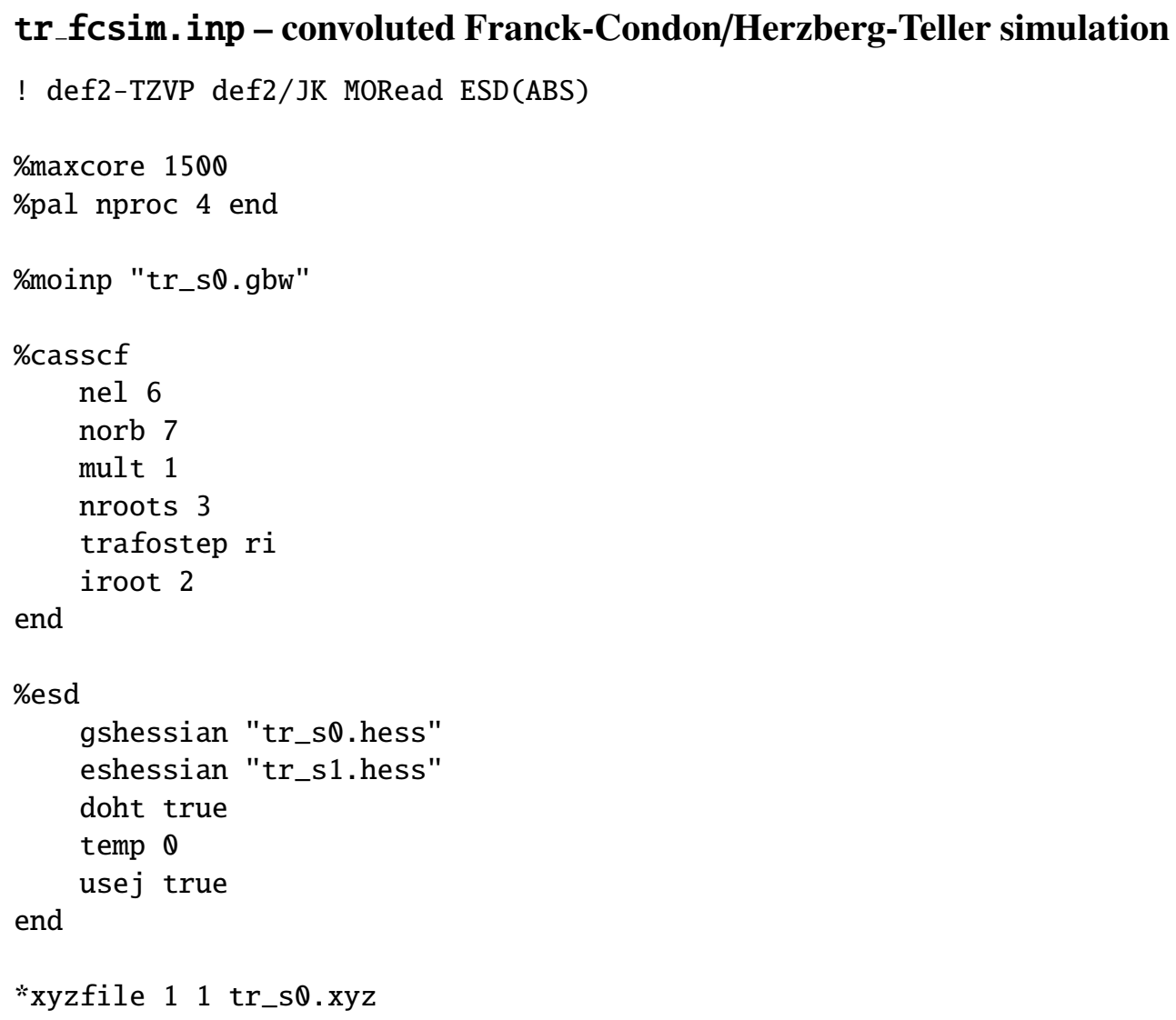




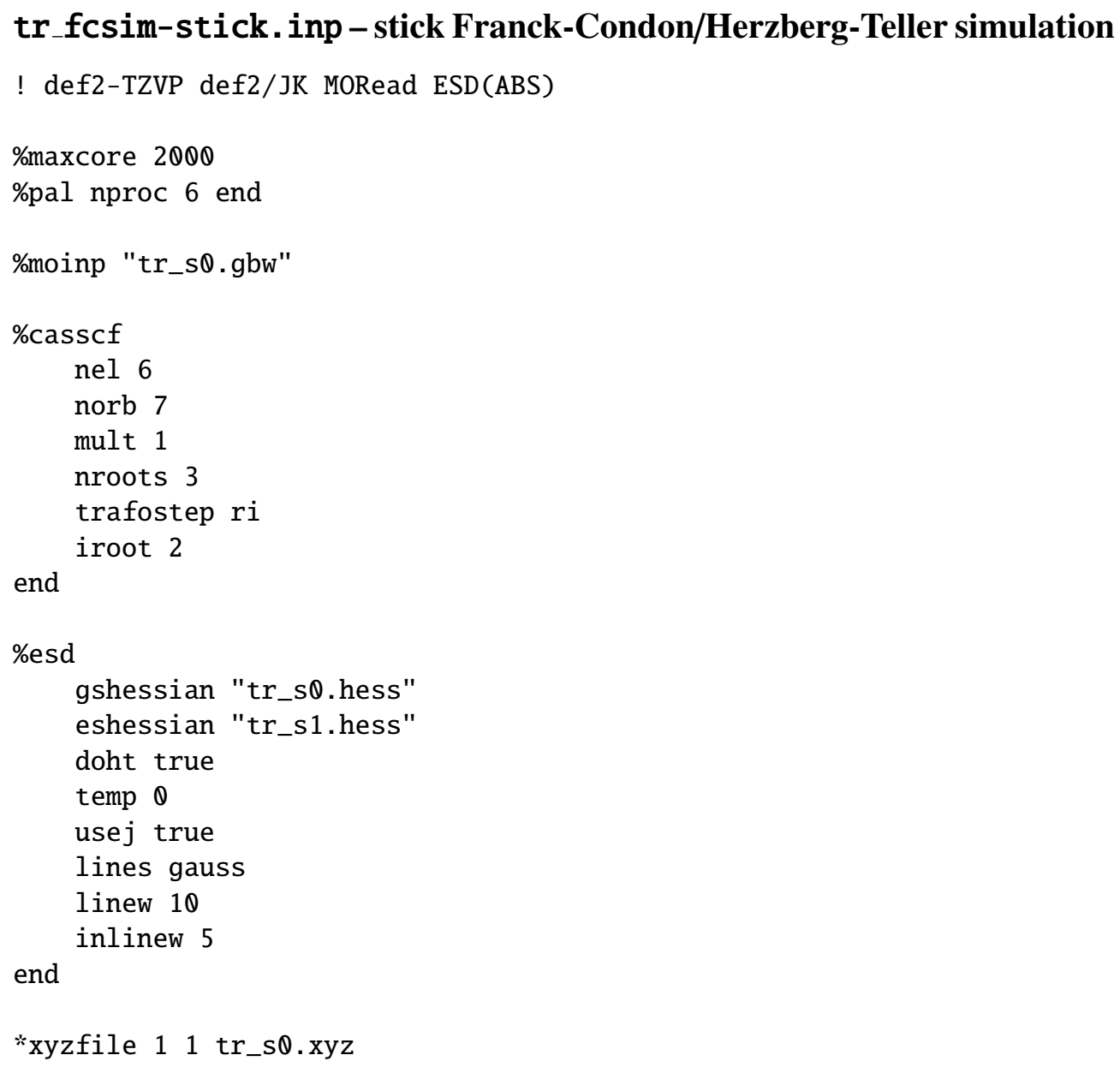


tr_s1scan.inp - Potential energy surface scan along $v 2$ and $v 7$ normal coordinates

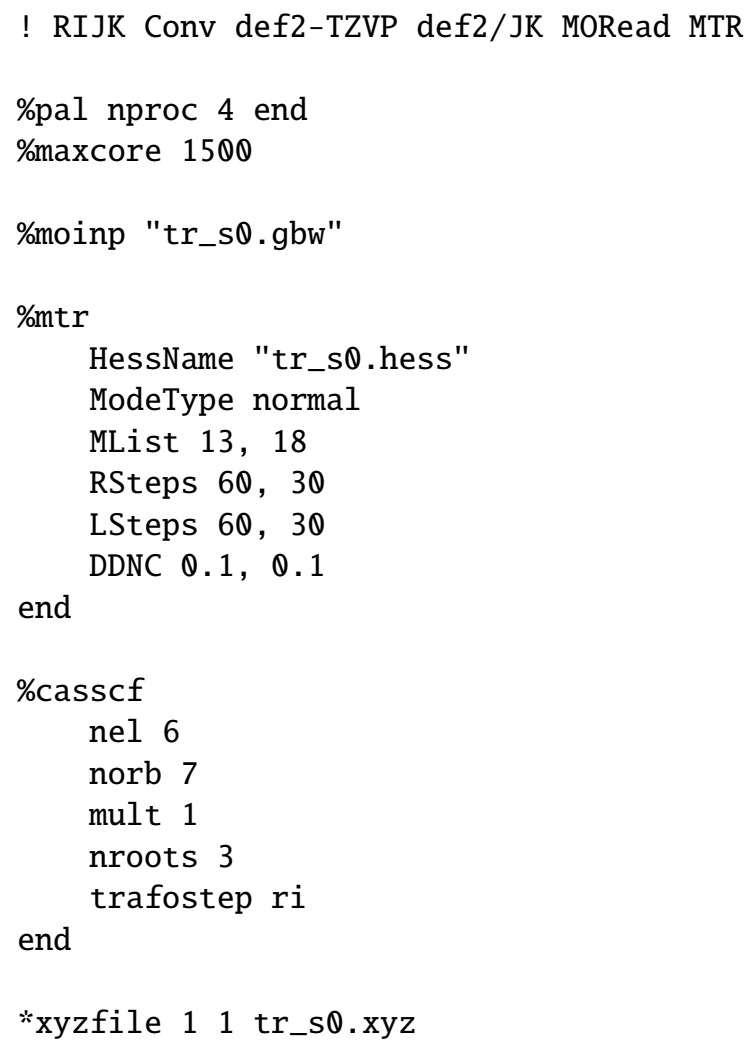


tr_s1scan.inp - Potential energy surface scan along the two degenerate $v 7$ normal coordinates

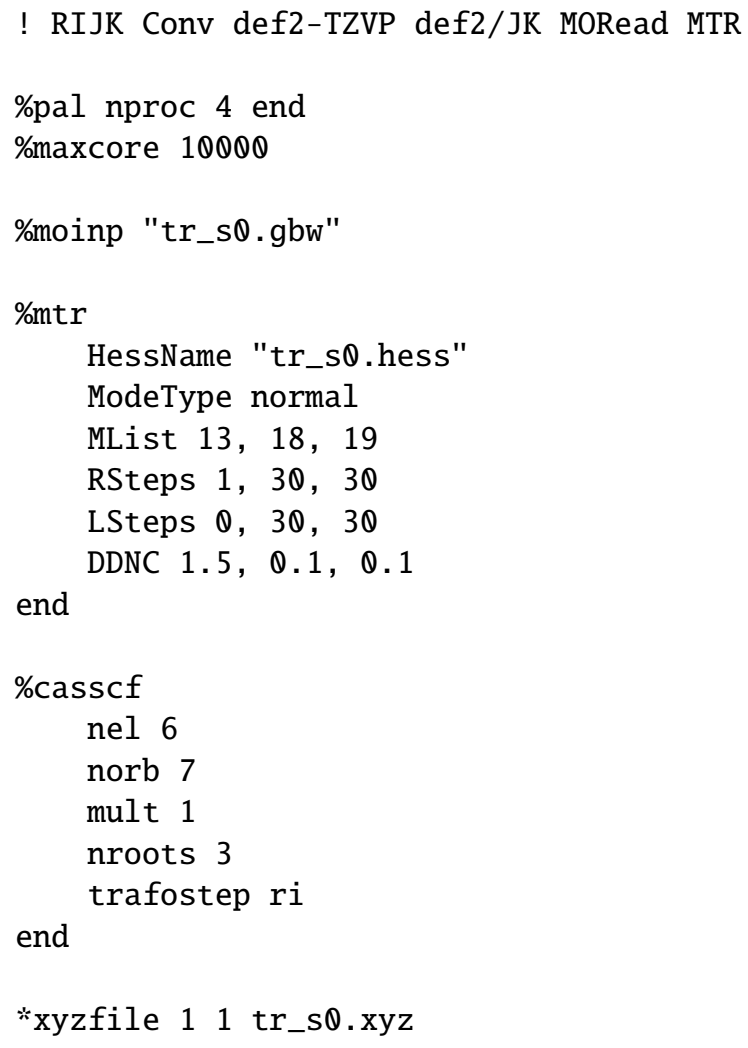




\section{References}

[1] Zhao, Y.; Truhlar, D. G. Design of Density Functionals that are Broadly Accurate for Thermochemistry, Thermochemical Kinetics, and Nonbonded Interactions. J. Phys. Chem. A 2005, 109, 5656-5667.

[2] Grimme, S.; Ehrlich, S.; Goerigk, L. Effect of the Damping Function in Dispersion Corrected Density Functional Theory. J. Comp. Chem. 2011, 32, $1456-1465$.

[3] Papajak, E.; Zheng, J.; Xu, X.; Leverentz, H. R.; Truhlar, D. G. Perspectives on Basis Sets Beautiful: Seasonal Plantings of Diffuse Basis Functions. $J$. Chem. Theory Comput. 2011, 7, 3027-3034.

[4] Guo, Y.; Riplinger, C.; Becker, U.; Liakos, D. G.; Minenkov, Y.; Cavallo, L.; Neese, F. An Improved Linear Scaling Perturbative Triples Correction for the Domain Based Local Pair-Natural Orbital Based Singles and Doubles Coupled Cluster Method [DLPNO-CCSD (T)]. J. Chem. Phys. 2018, 148, 011101.

[5] Kendall, R. A.; Dunning Jr, T. H.; Harrison, R. J. Electron Affinities of the First-Row Atoms Revisited. Systematic Basis Sets and Wave Functions. $J$. Chem. Phys. 1992, 96, 6796-6806.

[6] Frisch, M. J. et al. Gaussian 16 Revision B.01. 2016; Gaussian Inc. Wallingford CT.

[7] Neese, F. Software Update: the ORCA Program System, Version 4.0. WIREs Comput. Mol. Sci. 2018, 8, e1327.

[8] Wenthold, P. G.; Hrovat, D. A.; Borden, W. T.; Lineberger, W. C. TransitionState Spectroscopy of Cyclooctatetraene. Science 1996, 272, 1456-1459.

[9] Stanton, J. F.; Bartlett, R. J. The Equation of Motion Coupled-Cluster Method. A Systematic Biorthogonal Approach to Molecular Excitation Energies, Transition Probabilities, and Excited State Properties. J. Chem. Phys. 1993, 98, 7029-7039.

[10] de Souza, B.; Neese, F.; Izsák, R. On the Theoretical Prediction of Fluorescence Rates from First Principles using the Path Integral Approach. J. Chem. Phys. 2018, 148, 034104.

[11] Roos, B. O. The Complete Active Space Self-Consistent Field Method and its Applications in Electronic Structure Calculations. Advances in Chemical Physics: Ab Initio Methods in Quantum Chemistry Part 2 1987, 69, 399-445. 
[12] Weigend, F.; Ahlrichs, R. Balanced Basis Sets of Split Valence, Triple Zeta Valence and Quadruple Zeta Valence Quality for H to Rn: Design and Assessment of Accuracy. Phys. Chem. Chem. Phys. 2005, 7, 3297-3305.

[13] Weigend, F. Hartree-Fock Exchange Fitting Basis Sets for H to Rn. J. Comput. Chem. 2008, 29, 167-175.

[14] Longuet-Higgins, H. C.; Öpik, U.; Pryce, M. H. L.; Sack, R. A. Studies of the Jahn-Teller Effect. II. The Dynamical Problem. Proc. Roy. Soc Lond. 1958, $244,1-16$.

[15] Child, M. S.; Longuet-Higgins, H. C. Studies of the Jahn-Teller effect. III. The Rotational and Vibrational Spectra of Symmetric-Top Molecules in Electronically Degenerate States. Phil. Trans. Roy. Soc. 1961, 254, 259-294.

[16] Cossart-Magos, C.; Cossart, D.; Leach, S. Emission Spectra of Seven Fluorobenzene Cations: Dynamical Jahn-Teller effect in sym- $\mathrm{C}_{6} \mathrm{~F}_{3} \mathrm{H}_{3}^{+}$and $\mathrm{C}_{6} \mathrm{~F}_{6}^{+}$. Mol. Phys. 1979, 37, 793-830.

[17] Applegate, B. E.; Barckholtz, T. A.; Miller, T. A. Explorations of Conical Intersections and their Ramifications for Chemistry through the Jahn-Teller effect. Chem. Soc. Rev. 2003, 32, 38-49. 